\title{
Vibrational Sum Frequency Generation (VSFG) Spectroscopy Measurement of the Rotational Barrier of Methyl Groups on Methyl- Terminated Silicon(111) Surfaces
}

\section{Supporting Information}

Dhritiman Bhattacharyya, ${ }^{\dagger}$ Angelo Montenegro, ${ }^{\dagger}$ Noah T. Plymale,,${ }^{\ddagger}$ Chayan Dutta, ${ }^{\dagger}$ Nathan S. Lewis, $*, \$$ Alexander V. Benderskii ${ }^{*} \dagger$

${ }^{\dagger}$ Department of Chemistry, University of Southern California, Los Angeles, California 900890482, United States

$\$$ Division of Chemistry and Chemical Engineering, and Beckman Institute, California Institute of Technology, Pasadena, California 91125, United States

* Corresponding author: nslewis@its.caltech.edu

* Corresponding author: alex.benderskii@usc.edu

\section{Experimental set-up:}

A dual-stage Ti-Sapphire amplifier system operating at a $5 \mathrm{kHz}$ repetition rate and generating a femtosecond $800 \mathrm{~nm}$ pulse with a pulse energy of $3 \mathrm{~mJ}$ was used to generate the light pulses for VSFG spectroscopy. One portion of the amplifier output (pulse width of $\sim 40$ fs and pulse energy of $1.5 \mathrm{~mJ}$ ) as used to pump the TOPAS optical parametric amplifier (OPA). The outputs from the OPA (signal and idler) were routed to the noncollinear difference frequency generator (NDFG) to generate a broadband mid-infrared pulse centered around $3000 \mathrm{~cm}^{-1}$ with a bandwidth of 250 $\mathrm{cm}^{-1}$. The other portion of the amplifier output was first externally compressed using a grating compressor and then passed through a $4 \mathrm{f}$ stretcher to narrow the pulse in frequency domain and produce a boxcar spectral profile. The frequency resolution was achieved by this picosecond 800 $\mathrm{nm}$ up-conversion pulse (FWHM $\sim 8-10 \mathrm{~cm}^{-1}$ ). At the sample stage, the power of the IR light 
was $4 \mu \mathrm{J} /$ pulse and that of the visible light was $7 \mu \mathrm{J} /$ pulse. The spot-size of the IR and the visible beams were $190 \mu \mathrm{m}$ and $230 \mu \mathrm{m}$ respectively. The details of the entire experimental set-up have been provided previously. ${ }^{1,2}$ The angle of incidence of the visible and the IR beam was $67^{0}$ and $62^{0}$ with respect to the surface normal. The SFG spectra were recorded using a liquid-nitrogencooled CCD detector $(2048 \times 512$ pixels). The PPP and SPS spectra were collected for $3 \mathrm{~min}$ and $10 \mathrm{~min}$, respectively. Both spectra were normalized to $1 \mathrm{~min}$ before fitting and comparing the respective line shapes. The fitting parameters are presented in Tables S1 and S2.

Table S1. Fitting parameters of the VSFG spectra for SPS polarization at five different temperatures.

\begin{tabular}{|c|l|l|l|l|l|}
\hline \multirow{2}{*}{} & \multicolumn{5}{|c|}{ Temperature $\left({ }^{\circ} \mathrm{C}\right)$} \\
\cline { 2 - 6 } & \multicolumn{1}{|c|}{$21^{0} \mathrm{C}$} & $38^{\circ} \mathrm{C}$ & $56^{0} \mathrm{C}$ & $85^{\circ} \mathrm{C}$ & $118^{0} \mathrm{C}$ \\
\hline$A_{N R}$ & $16.3 \pm 0.2$ & $20.6 \pm 0.2$ & $19.9 \pm 0.2$ & $19.7 \pm 0.3$ & $19.9 \pm 0.3$ \\
\hline$\varphi$ & $1.3 \pm 0.1$ & $1.5 \pm 0.1$ & $1.5 \pm 0.1$ & $1.7 \pm 0.2$ & $1.9 \pm 0.2$ \\
\hline$B$ & $-39.5 \pm 4.0$ & $-52.2 \pm 6.9$ & $-56.8 \pm 6.6$ & $-63.2 \pm 8.5$ & $-75.1 \pm 12.5$ \\
\hline$\omega$ & $2980.5 \pm 1.3$ & $2976.8 \pm 1.7$ & $2975.5 \pm 1.7$ & $2972.3 \pm 1.9$ & $2968.8 \pm 2.5$ \\
\hline$\Gamma$ & $13.5 \pm 1.1$ & $16.2 \pm 1.5$ & $17.0 \pm 1.3$ & $17.6 \pm 1.4$ & $18.4 \pm 1.6$ \\
\hline
\end{tabular}

Table S2. Fitting parameters of the VSFG spectra for PPP polarization at five different temperatures.

\begin{tabular}{|c|c|c|c|c|c|}
\hline \multirow{2}{*}{} & \multicolumn{5}{|c|}{ Temperature $\left({ }^{\circ} \mathrm{C}\right)$} \\
\cline { 2 - 6 } & $21^{0} \mathrm{C}$ & $38^{0} \mathrm{C}$ & $56^{0} \mathrm{C}$ & $85^{\circ} \mathrm{C}$ & $118^{0} \mathrm{C}$ \\
\hline$A_{N R}$ & $41.8 \pm 0.7$ & $46.8 \pm 1.2$ & $44.3 \pm 1.4$ & $44.0 \pm 1.3$ & $48.4 \pm 1.2$ \\
\hline$\varphi$ & $19.7 \pm 0.1$ & $20.0 \pm 0.1$ & $20.0 \pm 0.1$ & $20.1 \pm 0.1$ & $20.2 \pm 0.1$ \\
\hline$B_{1}$ & $-251.3 \pm 14.6$ & $-295.8 \pm 26.4$ & $-370.9 \pm 31.5$ & $-465.4 \pm 28.4$ & $-488.0 \pm 28.1$ \\
\hline$\omega_{1}$ & $2915.4 \pm 0.4$ & $2911.4 \pm 0.5$ & $2911.5 \pm 0.5$ & $2909.4 \pm 0.4$ & $2908.5 \pm 0.4$ \\
\hline$\Gamma_{1}$ & $12.5 \pm 0.5$ & $13.1 \pm 0.8$ & $14.1 \pm 0.7$ & $13.7 \pm 0.5$ & $13.9 \pm 0.5$ \\
\hline
\end{tabular}




\begin{tabular}{|c|c|c|c|c|c|}
\hline$B_{2}$ & $-315.3 \pm 21.6$ & $-267.8 \pm 29.1$ & $-306.9 \pm 33.1$ & $-341.2 \pm 27.2$ & $-291.8 \pm 21.7$ \\
\hline$\omega_{2}$ & $2974.1 \pm 0.4$ & $2968.9 \pm 0.5$ & $2968.9 \pm 0.5$ & $2966.8 \pm 0.4$ & $2965.3 \pm 0.4$ \\
\hline$\Gamma_{2}$ & $11.6 \pm 0.5$ & $12.1 \pm 0.8$ & $12.3 \pm 0.8$ & $12.0 \pm 0.6$ & $10.7 \pm 0.5$ \\
\hline
\end{tabular}

\section{References}

1. Vinaykin, M.; Benderskii, A. V., Vibrational sum-frequency spectrum of the water bend at the air/water interface. J. Phys. Chem. Lett. 2012, 3 (22), 3348-3352.

2. Bhattacharyya, D., Montenegro, A., Dhar, P., Mammetkuliyev, M., Pankow, R.M., Jung, M.C., Thompson, M.E., Thompson, B.C. and Benderskii, A.V., Molecular Orientation of Poly-3-Hexylthiophene at the Buried Interface with Fullerene.J. Phys. Chem. Lett. 2019, 10 (8), 1757-1762 